\title{
IMPROVING THE PERFORMANCE PARAMETERS OF MICROSTRIP PATCH ANTENNA BY USING EBG SUBSTRATE
}

\author{
Gaurav Kumar Sharma ${ }^{1}$, Narinder Sharma ${ }^{2}$ \\ ${ }^{I}$ M.Tech. Scholar, Department of ECE, Amritsar College of Engineering and Technology AMRITSAR, PUNJAB, \\ INDIA, gaurav.bhanot@yahoo.com \\ 2 Associate Professor, Department of EEE, Amritsar College of Engineering and Technology AMRITSAR, PUNJAB, \\ INDIA, narinder.acet@gmail.com
}

\begin{abstract}
The objective of this paper is to analyze the performance of electromagnetic band gap (EBG) antenna for base station applications through simulation. The proposed analysis is carried out using the high frequency structure simulator (HFSS). In our method, to overcome several intrinsic limitations of patch antennas such as constrict bandwidth, low gain, excitation of surface waves, the EBG concept is applied. The patch antenna and patch antenna surrounded by the EBG cells are characterized in terms of return loss and radiation pattern in an anechoic chamber.
\end{abstract}

Index Terms: Microstrip patch antenna, electromagnetic band gap structure, bandwidth, gain.

\section{INTRODUCTION}

With the rapid development of wireless field, microstrip antennas became more appealing in antenna community. These antennas are low-profile, low price and dismount. However, Surface waves are a major drawback for this type of antenna as they lower the antenna efficiency. A narrow impedance bandwidth is another limitation of patch antennas. Two techniques have been applied to suppress surface wave propagation, namely micromachining [1-2] and periodic structures called the electromagnetic band gap (EBG) structures [3-6]. In microstrip antennas, EBG structures environing patch element to suppress surface waves to attain better side lobe and back lobe levels and enhanced radiation efficiency. It has been shown in article [7] that, placing EBG cells in E-plane is enough for surface wave reducing and it is not required to have $H$-plane EBG cells. This EBG structures is used in microstrip array antennas between radiating array elements in $E$-plane to come down mutual coupling between them by suppressing surface waves. However, the consequences of EBG structures environing the antenna can be considered as two consequences, namely parasitic loading effect and cavity effect. The parasitic loading effects enhance the bandwidth, whereas the cavity effect diminishes the bandwidth. The EBG cells placed in nearness of the radiating edges of the antenna components provide the parasitic loading and thereby bettered bandwidth [8]. On the other hand, the EBG structures also reflect back a part of the energy that circulate along the substrate of the antenna, thus acting as reflecting walls across the antenna and thereby the cavity effect. With elite rows of EBG structures, minus energy is reflected back and the parasitic effect becomes prevailing. This contributes to the significant enhancement in the bandwidths. As the number of rows is increased, more of the energy circulate along the substrate is reflected back and the cavity effect becomes prevailing. This in turn enhances the Q-factor of the cavity made by the EBG structures surrounding the antenna and come down the bandwidths of the antenna. On the other hand, front to back ratio (FBR) of the antenna enhances with the number of EBG rows surrounding the antenna, as anticipated, due to the suppression of surface waves.

Since antenna radiation features, incubate resonant frequency and radiation patterns are ascertained for the most part by its shapes and size, altering its geometry mechanically allow for a non-rational method to reconfigure its radiation attributes. MEMS techniques incorporate electrical and mechanical operates in a single component using micro fabrication or micro aching technology, and can be recognized with present semiconductor integrated circuit serving [5-7]. Hence, MEMS based reconfigurable antennas had been widely inquired [8-13]. By using printed circuit board (PCB) techniques, planar wire antennas in the form of filamentary conductive traces can be constructed with inserted MEMS switches. A MEMS switch converts dynamically the antenna length and enables dual or multiband applications.

Antenna reconfiguration can also be projected electronics switch such as PIN diodes, which are ordinarily used in modern communications and radar applications. When the diode switch is DC forward biased, it is at an "on" state and is ideally a short circuit; while when the diode is reverse biased, it is at an "off" state and the diode is open-circuited. Placing a PIN diode in between two conducting traces, could control electronically the efficient antenna physical length. Planar microstrip antennas using PIN diode switching for reconfiguration had also been investigated [48].

Most reconfigurable antennas are grounded on the dynamic control of the antenna physical length. It is possible to design electronically tunable antennas by adapting the attributes of the material where an antenna occupies. Ferrite- 
Loaded microstrip antennas are examples [2], where the antenna features are aligned by the external magnetic fields issued by a de biased circuit.

Electromagnetic band gap (EBG) structures in printed circuits are thin complex dielectric layers with periodic metallic patterns (generally backed by a metal ground plane), and have one or multiple frequency band-gaps in which no substrate mode can exist. This unique ownership has been utilized to design antenna systems with a improve gain and efficiency, lower side-lobes and back-lobe levels amend isolations within array elements, by crushing surface wave modes [2-4]. The EBG substrate has been utilized to alleviate some withdraws of conventional microstrip antennas [5-7]. Recently, radiation features of a microstrip patch over a mushroom-type electromagnetic band-gap (EBG) substrate were inquired [9]. The EBG surface e is found to have the impressions of diluting the patch resonant length and bandwidth as a result of stronger capacitive coupling.

In this paper, the functioning of a rectangular microstrip patch antenna has been mended utilizing a new cylindrical electromagnetic band-gap (EBG) substrate. The patch antenna is fed by a driven terminal and is integrated within a cylindrical electromagnetic band-gap substrate, based on the mushroom-like substrate, to raise the antenna gain and bandwidth. The cylindrical electromagnetic band-gap structure applied is a combination of two periodic structures with dissimilar periods. The feature of the patch antenna based on drilling air holes and embedding a metal boundary in the substrate were studied by [9]. The outcome suggested that the surface waves which spread along the surface of the substrate can be inhibited by the multiple photonic band-gap structure because of its consequences of forbidden band, that it can diversify almost of electromagnetic waves' energy in the substrate importantly, and that it has lower return loss (S11) compared to the conventional patch antennas and enhanced gain.

\section{ELECTROMAGNETIC BAND-GAP STRUCTURE}

Periodic structures are ample in cosmos beguiled artists and scientists alike. When an electromagnetic waves energizing procedures amazing boa
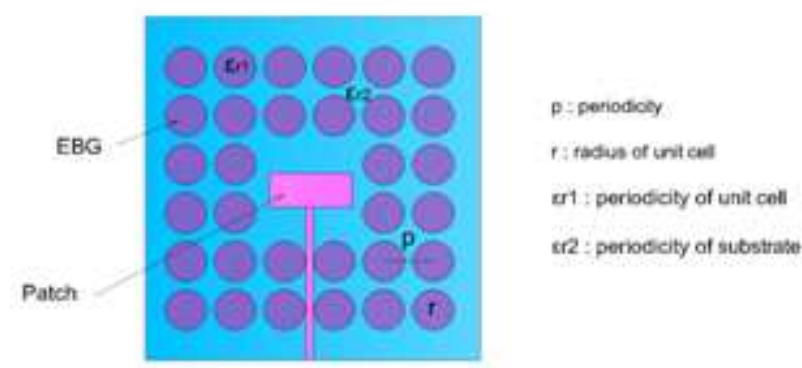

Fig 1: Electromagnetic band-gap fundamental structure showing radius and periodicity of structure
In detail, boats frequency stop-bands, pass-bands, and band identified [1]. Brushing up the literature, on several nomenclatures have been applied looking of the applications. These applications are innovation, gratings, frequency exclusive surface photonic crystals [2] and photonic band-gaps They can be assorted beneath the liberal Electromagnetic Band-gap (EBG) structures in General speaking, electromagnetic banish are specified as unreal periodic (or formerly objects that forbid/aid the propagation of waves in a assigned band of frequency for al and all polarization states (as shown in $\mathrm{F}$ structures are commonly recognized by periodic dielectric materials and metallic conductors (as shown in fig. 1). In universal, they can be categorized in allowing to their geometric configuration: (1) dimensional volumetric structures, (2) two-dimensional surfaces, and (3) one-dimensional.

\section{ANALYSIS AND DESIGN}

In this division, a rectangular microstrip patch antenna and its cylindrical EBG substrate are projected by applying a Finite Element method (HFSS-Ansoft). In the pretense operation convergence and minimization of numerical errors were received by assuring that the mesh was sufficiently alright. In Subsection A, the patch antenna is planned to function at an arbitrary chosen frequency. Then, in Subsection B, the EBG substrate is projected to enhance the gain of the patch antenna at its functioning frequency.

\subsection{Rectangular microstrip patch antenna}

This subsection identifies the patch-antenna configuration that is chose to execute the comparison between using a normal substrate and a cylindrical EBG substrate. The antenna is depicted in Fig. 2. The patch antenna is rectangular with length of $1=15.8 \mathrm{~mm}$ and breath is $\mathrm{b}=$ $8 \mathrm{~mm}$ printed on a substrate with a relative permittivity of or $=2.2$. The patch is fed by a probe and placed $6 \mathrm{~mm}$ from the center of the patch. The feed location was optimized to afford better impedance matching. The sizing of the substrate is $180 \mathrm{~mm} \times 180 \mathrm{~mm}$. The antenna resonates at $10.667 \mathrm{GHz}$.

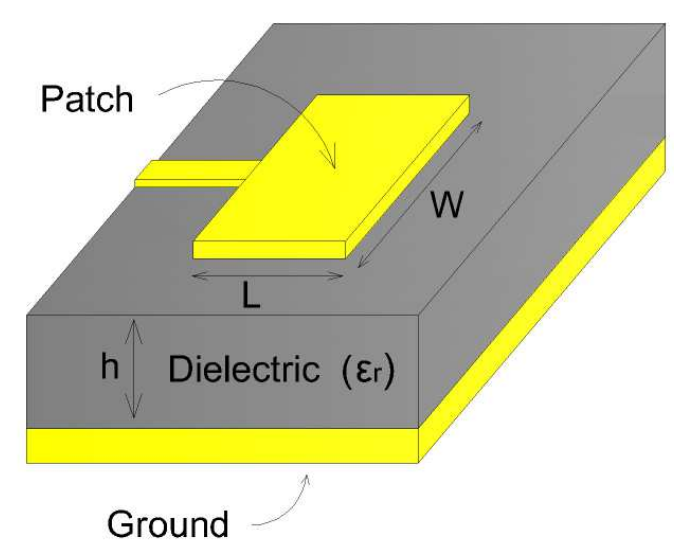

Fig 2: Rectangular patch antenna with microstrip. 
The maximum gain of the patch antenna is about $6 \mathrm{~dB}$. The gain of this antenna is heightening using a cylindrical EBG substrate, which is depicted in the next subsection.

\subsection{Patch antenna surrounded by a cylindrical EBG substrate}

While the previous subsection has defined the patch-antenna configuration, the design of the EBG substrate is now presented. Figure 3 shows the schematic of the proposed patch antenna surrounded by a cylindrical structure composed of metal rings and grounding vias. The concentric rings of strips are etched on the same plane than the patch antenna, with the distance $5 \mathrm{~mm}$ from one to another. The first metal ring starts at the radius $2 \mathrm{~mm}$.

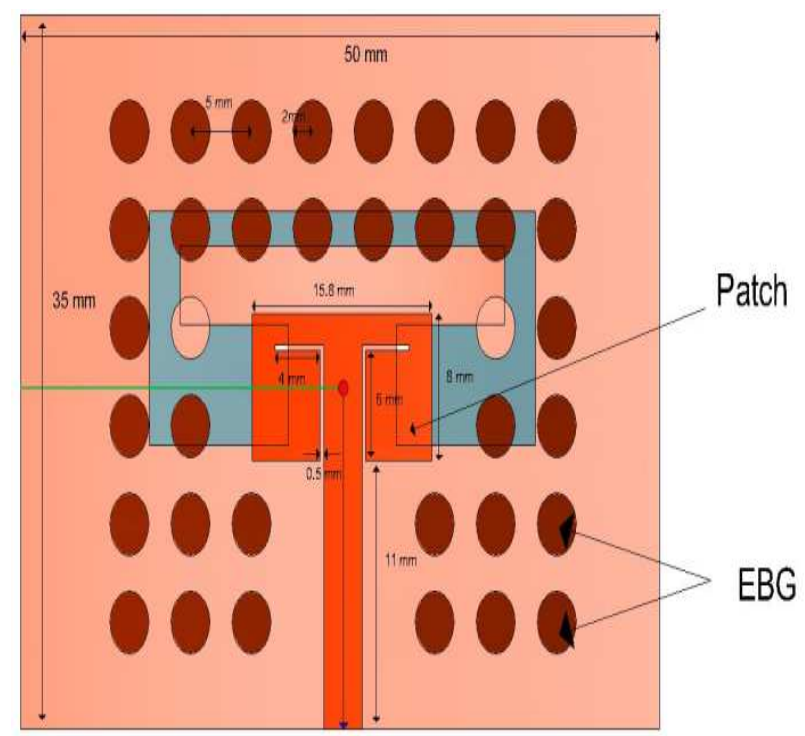

Fig 3: Patch incorporated with the cylindrical EBG substrate.

The vias have the radius a, and they are disposed with the same transversal period $\mathrm{Pt}$ and the same radial period Pr2.The following parameters are fixed: $2 \mathrm{a}=\mathrm{g}=2 \mathrm{~mm}$. The remaining parameters $\operatorname{Pr} 1$ and $\operatorname{Pr} 2$ were optimized. Three concentric rings and three circularly periodic structures of vias are considered. According to numerical results (not shown here), the gain is not sufficiently increased when only one or two layers are used.

\section{SIMULATION RESULT}

The proposed EBG-based antenna structure has been simulated using cell size $129 \times 131 \times 34$. Here, time step $\Delta \mathrm{t}$ $=2.0747 \mathrm{~ns}$. Results have been analyzed for variation of bandwidth, active $\mathrm{z}$ parameter, vswr, gain and reflection loss because EBG structures are expected to increase bandwidth and gain by suppression of surface waves. fig. 5 shows active $\mathrm{z}$ parameter got from the designed EBG structure. fig.4 shows bandwidth obtained for the proposed structure on rectangular patch. The vswr of the proposed design is shown in Fig.6.

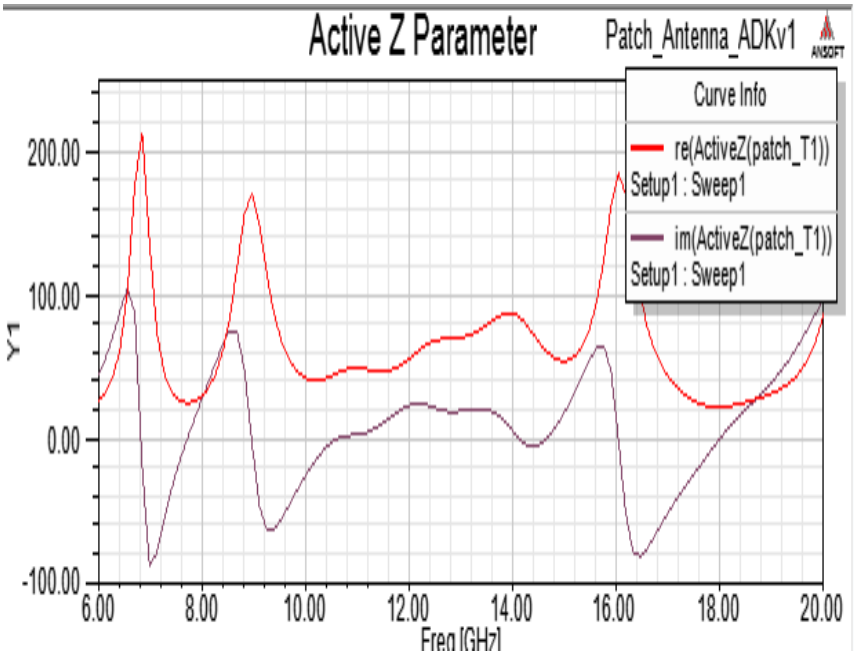

Fig 4: Z Parameter vs. frequency (in GHz) for the proposed structure.

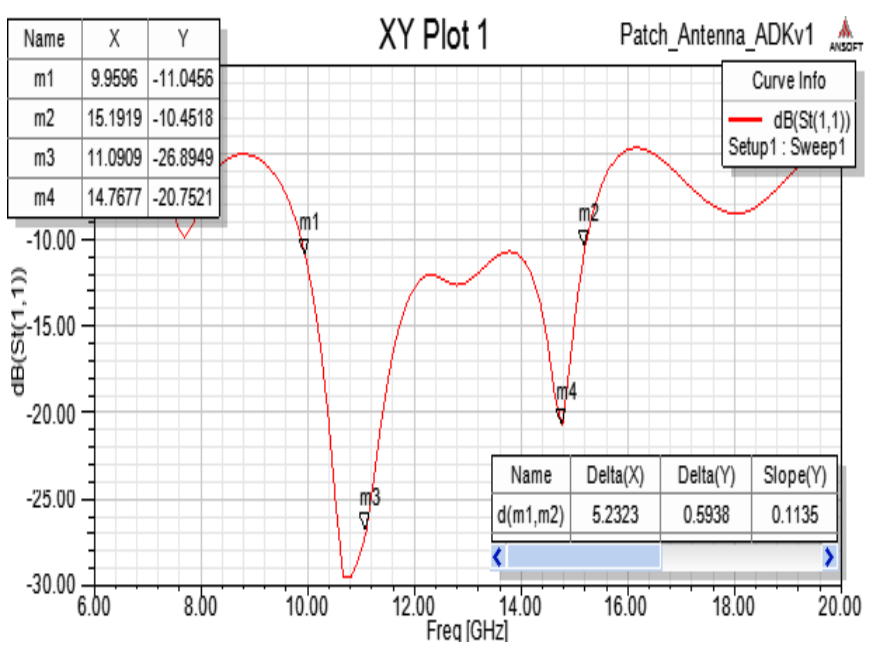

Fig 5: Z Bandwidth vs. frequency (in GHz) for the proposed structure.

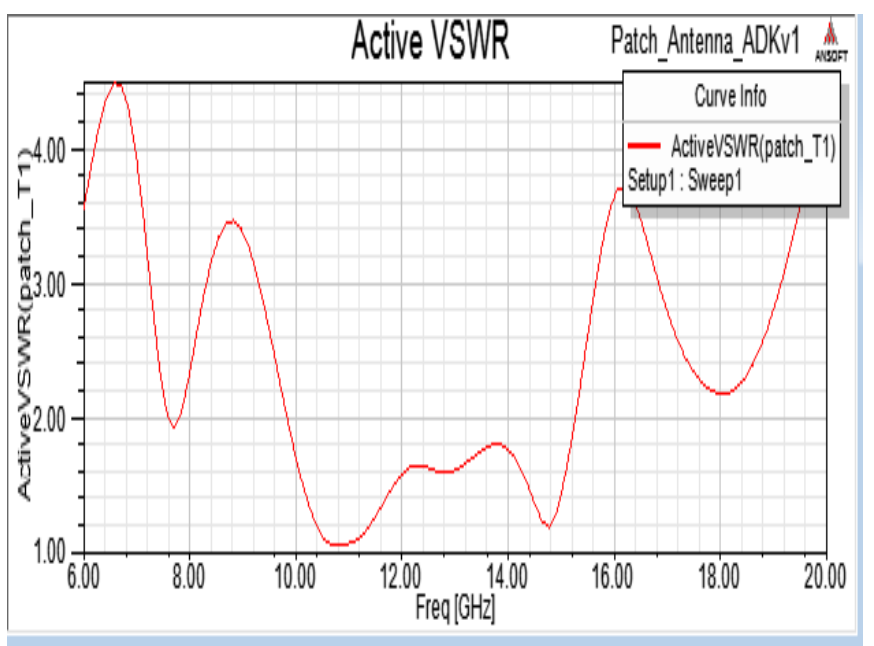

Fig 6: VSWR (in dB) vs. frequency (in GHz) for the proposed structure. 


\section{OBSERVATIONS}

Observations concerning the bandwidth, active $\mathrm{z}$ parameters, active vswr, and gain and reflection loss using the proposed antenna structure have been talked about and amended in this section.

\subsection{BANDWIDTH}

This design achieved an impedance bandwidth of $10.14 \%$ (2.34-2.59 GHz) at $-10 \mathrm{~dB}$ return loss. Simulated radiation pattern is almost omnidirectional. The simulated results prove the compatibility of the EBG antenna with the 2.46 $\mathrm{GHz}$ ISM band applications. Further enhancement of the antenna performance with improved design is under consideration.

\subsection{Gain}

Gain has been accounted from the radiation patterns in $\mathrm{E}$ and H-plane for the patch antenna. Gain receive from proposed antenna structure (EBG substrate) is found to be $7.7324 \mathrm{~dB}$ at $10.667 \mathrm{GHz}$ (from Fig. 5-6). Gain receive from patch without any EBG structures show a value of $6 \mathrm{~dB}$ at an operating frequency of $10.667 \mathrm{GHz}$. Gain obtained using proposed structure is importantly more than that without EBG.

\subsection{Reflection loss}

Reflection loss for aimed EBG-substrate patch is found to attain a lowest value of $-33 \mathrm{~dB}$. It is evident from the reflection loss curves of the antenna structures using EBG and that without EBG that reflection loss has reduced drastically for patch with EBG. This has ensued in a noteworthy improvement in gain. The bandwidth of the constitute EBG-based patch is bumped to be $112.3 \mathrm{MHz}$.

\section{DISCUSSION}

It is known that the bandwidth of the antenna can be enhanced by contracting any loss of the antenna. Selection of an effective quality (low-loss) substrate will contract the dielectric and conductor losses. However, surface waves go inside the substrate and reduce the bandwidth. To reduce this surface wave loss, wave propagation has to be stopped inside or reflected outside the substrate to enhance the bandwidth in forward direction. The waves are arrested or retarded by obstructing their path with a high propagationimpedance - air $(\varepsilon r=1)$. The impedance offered by a dielectric is given as $\eta / \sqrt[2]{E_{r}}$. This implies that the impedance offered, is maximum for smallest value of $\varepsilon r$ (the smallest value of $\varepsilon r$ being 1 for air). In the proposed antenna, the surface waves are reduced by drilling holes in periodic fashion in the dielectric substrate. The remarkable improvement in gain can be attributed to the periodic effect of embedding air voids (holes) in the substrate.

\section{CONCLUSION}

In this paper, a novel proficiency for the bandwidth sweetening of micro-strip patch antennas using a cylindrical EBG structure has been aimed and established. The EBG structure is based on the mushroom-like structure with a circular proportion. It is framed of a periodic structure of metallic rings and of a periodic structure of vias. The periods of time of these structures have been optimized applying a full-wave method to maximize the bandwidth. The new substrate comes down the surface wave, but the bandwidth enhancement is primarily due to the coupling between the patch and the EBG structure.

\section{REFERENCES}

[1] Gautheir, G. P., A. Courtay, and G. M. Rebiez, IMicrostrip antennas on synthesized low dielectric constant substrate," IEEE Trans. Ant. Propag., 1997.

[2] Papalymerou, I., R. F. Drayton, and L. P. B. Katehi, Micromachined patch antennas," IEEE

[3] Qian, Y., R. Coccioli, D. Sievenpiper, V. Radisic, E. Yablonovich, and T. Itoh, Microstrip patch antenna using novel photonic bandgap structures," Microw. J., Vol. 42, No. 1, 66\{76, 1999.

[4] M. Khayat, J. T. Williams, D. R. Jakson, and S. A. Long, "Mutual coupling between reduced surface-wave microstrip antennas, IEEE Trans. Antennas Propag., vol.48, pp. 15811593, Oct. 2000.

[5] D.M. Kokotoff, R.B. Waterhouse, C.R. Britcher, and J.T. Aberle, "Anular ring coupled circular patch with enhanced performance", Electron. Letters, vol.33, pp. 2000-2001, Nov. 1997.

[6] Agi, K., K. J. Maloy, E. Schmiloglu, M. Mojahedi, and E. Niver, Integration of a microstrip

patch antenna with a two-dimentional photonic crystal substrate," Electromagnetics, Vol. 19, 277\{290, May\{Jun. 1999.

[7] A. K. Bhattacharayya, "Characteristics of space and surfacewaves in a multilayered structure", IEEE Trans. Antennas Propag., vol.38, pp. 1231-1238, Aug. 1990..

[8] J. Joannopoulos, R. D. Meade and J. N. Winn, Photonic crystals: molding the flow of light, Princeton University Press, 1995M.

[9] R. Gonzalo, P. de Maagt, and M. Sorolla, "Enhanced pathantenna performance by supresing surface waves using photonic-bandgap substrates", IEEE Tran. Microw. Theo. Techn., vol. 47, pp. 2131-2138, Nov. 1999.

[10] D. Sievenpiper, L. Zhang, R.F. Jimenez Broas, N. G. Alexopoulos, and E. Yablonovitch, "High-impedance electromagnetic surfaces with a forbidden frequency band", IEEE Trans. Microwave Theory Techn., vol. 47, pp. 20592074, Nov. 1999.

[11] Y.-J. Park, A. Herchlein, and W. Wiesbeck, “A Photonic Bandgap (PBG) structure for guiding and suppressing surface waves in millimeter-wave antennas", IEEE Trans. Antennas Propagat., vol. 49, pp. 1854-1857, Oct. 2001.

[12] K. Agi, M. Mojahedi, B. Minhas, E. Schamilogu, and K. J. Malloy, "The effects of an electromagnetic crystal substrate 
on a microstrip patch antenna”, IEEE Trans. Antennas Propag., vol. 50, pp. 451-456, April 2002.

[13]F. Yang and Y. Rahmat-Samii, "Microstrip antennas integrated with electromagnetic bandgap (EBG) structures: a low mutual coupling design for array applications", IEEE Trans. Antennas Propag., vol. 51, pp. 2936-2946, oct. 2003.

[14] N. Llombart, A. Neto, G. Gerini, and P. de Maagt, "Planar circularly symettric EBG structures for reducing surface waves in printed antennas", IEEE Trans. Antennas Propag., vol. 53, pp. 3210-3218, Oct. 2005.

[15] A. Neto, N. Llombart, G. Gerini, and P. de Maagt, "On the optimal radiation bandwidth of printed slot antennas surrounded by EBGs", IEEE Trans. Antennas Propag., vol. 543, pp. 1074-1083, Ap. 2006.

\section{BIOGRAPHIES}

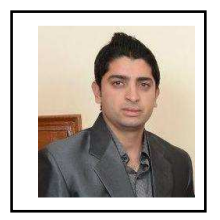

Gaurav Kumar Sharma, he is M.Tech (ECE) student at Amritsar college of Engineering \& technology, AMRITSAR. He has earlier completed her B.Tech in ECE from ACET, Amritsar. His area of interest are Antenna \& Wave Propagation, Antenna designing and fabrication, Signal Processing, and optical fiber communication. Email id: gaurav.bhanot@yahoo.com

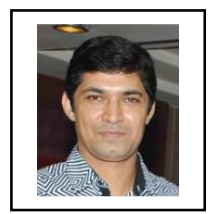

Narinder Sharma, he is working as HOD of EEE at Amritsar College of Engineering and Technology. He is B.Tech, M.Tech, and Qualified. He has attended many international and National conferences and have Published Paper in national and International Journals 